\title{
Tetrandrine induces G1/S cell cycle arrest through the ROS/Akt pathway in EOMA cells and inhibits angiogenesis in vivo
}

\author{
WENKAI XIAO $^{1 *}$, YAJIE JIANG ${ }^{1 *}$, QIUXU MEN ${ }^{1}$, LING YUAN $^{1}$, \\ ZEBO HUANG ${ }^{1}$, TING LIU², WENHUA LI ${ }^{2}$ and XIN LIU ${ }^{1}$ \\ ${ }^{1}$ Key Laboratory of Combinatorial Biosynthesis and Drug Discovery, Ministry of Education, \\ and School of Pharmaceutical Sciences; ${ }^{2}$ School of Life Science, Wuhan University, Wuhan, Hubei 430071, P.R. China
}

Received August 29, 2014; Accepted October 16, 2014

DOI: 10.3892/ijo.2014.2735

\begin{abstract}
Tetrandrine, a bisbenzylisoquinoline alkaloid, is known to inhibit tumor cell proliferation and induce apoptosis in cancer models in vitro and in vivo. In the present study, tetrandrine significantly inhibited the proliferation of mouse endothelial cells (EOMA cell) and induced G1/S arrest in EOMA cells, in which the expressions of cyclin D and cyclin $\mathrm{E}$ and CDKs were downregulated. Tetrandrine treatment also caused intracellular accumulation of reactive oxygen species (ROS). Pretreatment with NAC, which is a ROS inhibitor, blocked G1/S cell arrest and cyclin regulation induced by tetrandrine, implying that ROS generation plays an important role in tetrandrine-induced cell cycle arrest. Furthermore, a decreased phospho-Akt protein level after tetrandrine treatment was reversible with the removal of the intracellular ROS by NAC. Notably, overexpression of Akt decreased tetrandrineinduced G1/S arrest. Finally, we verified the antiangiogenic effects of tetrandrine in vivo in a liver cancer xenograft model in nude mice. In conclusion, tetrandrine inhibits EOMA cell growth through the ROS/Akt pathway, and it could be a promising compound for cancer therapy as an inhibitor of tumor vascular growth.
\end{abstract}

\section{Introduction}

Antiangiogenic therapy is an effective method of reducing tumor growth in animal models (1). Recently, several angio-

Correspondence to: Dr Xin Liu, School of Pharmaceutical Sciences, Wuhan University, 185 East Lake Road, Wuhan, Hubei 430071, P.R. China

E-mail: 1x@whu.edu.cn

*Contributed equally

Abbreviations: Tet, tetrandrine; ROS, reactive oxygen species; NAC, L-N-acetylcysteine; MTT, 3-(4,5-dimethylthiazol-2-yl)-2,5-diphenyl tetrazolium bromide; DCFH-DA, 2'-7'-dichlorodihydrofluorescein diacetate; PI3K, phosphatidylinositol 3-kinase

Key words: tetrandrine, G1/S arrest, reactive oxygen species, Akt, CD31, angiogenesis genesis inhibitors such as bevacizumab and endostatin have been applied in clinical therapy. In addition, a growing number of compounds have in recent years been discovered to inhibit tumor angiogenesis in laboratory cancer studies $(2,3)$. Notably, some traditional medicines in clinical applications are also found to have antiangiogenic properties (4).

Tetrandrine, a bisbenzylisoquinoline alkaloid originally isolated from the roots of the medicinal plant Stephaniae tetrandrae S. Moore (Han-Fang-Ji in Chinese), is found to have a variety of pharmacological properties, including antiallergic, anti-inflammatory and anticancer activities (5-7). For example, tetrandrine is shown to inhibit proliferation and induce apoptosis in vitro in hepatocellular, lung carcinoma, bladder cancer and colon carcinoma (8-13). Tetrandrine is able to increase the level of ROS and decrease the expression of glutathione, leading to the inhibition of tumor cell proliferation $(13,14)$. It is also reported that tetrandrine can induce cell cycle arrest and apoptosis in Hep G2 cells via the p53 and p21/WAF1 pathway (15). Previous studies also indicate that tetrandrine is a promising cancer therapy compound for its effect on angiogenesis $(6,16,17)$, however, little is known on the molecular mechanisms for the effects of tetrandrine in cell cycle and angiogenesis in endothelial cells and its function in the vessels.

The EOMA cell line is derived from a mixed hemangioendothelioma in an adult mouse and has a characteristic protein expression profile of endothelial cell phenotype such as high CD31 and CD45 expression (18-21). It is known that EOMA cells are widely used in the research field of angiogenesis inhibitors $(22,23)$. Endostatin, a clinically used anti-angiogenesis drug, has been shown to be effective on EOMA cells (24). In the present study, tetrandrine was able to cause G1/S cell cycle arrest by promoting excessive ROS accumulation in EOMA cells and downregulating expression of cyclins. We also show the anti-angiogenic effect in vivo of tetrandrine in liver tumors in nude mice.

\section{Materials and methods}

Chemicals and antibodies. Tetrandrine was purchased from Shanghai Ronghe Medical (Shanghai, China). 2'-7'-Dichlorodihydrofluorescein diacetate (DCFH-DA), was purchased from Invitrogen (Carlsbad, CA, USA). FuGene HD 
tranfection reagent was from Promega (Madison, WI, USA) and trypan blue dye was from Sigma (St. Louis, MO, USA). The ROS Assay kit and LY294002 were purchased from Beyotime (Nantong, China). Propidium iodide (PI) was purchased from MP Biomedicals (Solon, OH, USA). 3-(4,5-Dimethylthiazol2-yl)-2,5-diphenyltetrazolium bromide (MTT) was purchased from Amresco LLC (Solon, OH, USA). Antibodies against $\beta$-tubulin, cyclin D1, cyclin D2, cyclin D3, cyclin E1, cyclin E2, CDK2, CDK4, GSK-3 $\beta$, p53 and p27 were from Proteintech (Wuhan, China), Akt and phosphor-Akt were obtained from Cell Signaling Technology (Boston, MA, USA). Antibody against mouse CD31 was obtained from Becton-Dickinson (Franklin, NJ, USA).

Cell lines and vectors. EOMA cells were purchased from the American Type Culture Collection (ATCC, Manassas, VA, USA). Huh7 cells were purchased from the China Center for Type Culture Collection (CCTCC, Wuhan, China). All the cells were cultured at $37^{\circ} \mathrm{C}$ in a humidified atmosphere of $95 \%$ air and $5 \% \mathrm{CO}_{2}$ in high-glucose DMEM supplemented with $10 \%$ fetal bovine serum, $1 \%$ penicillin and $1 \%$ streptomycin. Cell culture dishes and plates were obtained from the NEST Biotechnology Co., Ltd., Jiangsu, China.

Empty vector (PUSE) and Akt overexpression vector (PUSE-CA-Akt) were obtained form Upstate Biotechnology (Lake Placid, NY, USA). Briefly, EOMA cells were seeded at a concentration of $5 \times 10^{4}$ cells/well in a 6 -well plate and grown at $37^{\circ} \mathrm{C}$ for $22 \mathrm{~h}$ before transfection. Then the cells were transiently transfected using FuGene HD transfection reagent with $4 \mu \mathrm{g}$ of PUSE or PUSE-CA-Akt, respectively. After transfection for $16-24 \mathrm{~h}$, cells were treated with $30 \mu \mathrm{M}$ tetrandrine for $48 \mathrm{~h}$.

Cell proliferation assay. EOMA cells were seeded at a concentration of $5 \times 10^{4}$ cells/well in a 6 -well plate and grown at $37^{\circ} \mathrm{C}$ for $22 \mathrm{~h}$. The effects of tetrandrine on cell proliferation were characterized by cell counting. In brief, various concentrations of tetrandrine solution were added to different wells for $48 \mathrm{~h}$. To assess cellular proliferation, the cells were counted daily using a hemocytometer.

Cell cycle analysis. EOMA cells were seeded in $60-\mathrm{mm}$ plates at a concentration of $5 \times 10^{5}$ cells/plate. After incubation for $22 \mathrm{~h}$, various concentrations of tetrandrine solution were added, and the cells were incubated for $48 \mathrm{~h}$. EOMA cells were harvested and supplemented with $70 \%$ ice-cold ethanol overnight. The cells were treated with RNase $(50 \mu \mathrm{g} / \mathrm{ml})$ at $37^{\circ} \mathrm{C}$ for $1 \mathrm{~h}$, and treated with PI $(20 \mu \mathrm{g} / \mathrm{ml})$ for $30 \mathrm{~min}$ without light at $4^{\circ} \mathrm{C}$. The DNA content was analysed by flow cytometry (Beckman Coulter).

Western blot analysis. EOMA cells were treated with different concentrations of tetrandrine for $48 \mathrm{~h}$ and harvested. Then, supernatant was collected, and the protein concentration was determined with a Bicinchoninic acid protein assay kit (Pierce). Protein samples were separated by sodium dodecyl sulphate polyacrylamide gel electrophoresis (SDS-PAGE) and transferred to a PVDF membrane. Following antibody incubation, enhanced chemiluminescence was used to detect the proteins.
Evaluation of ROS expression using fluorescence microscopy and flow cytometry. EOMA cells were washed twice with PBS. 2'-7'-Dichlorodihydrofluorescein diacetate (DCFH-DA) was diluted to $10 \mu \mathrm{M}$ with HG-DMEM and added to the cells. The plates were then stored in an incubator at $37^{\circ} \mathrm{C}$ for $1 \mathrm{~h}$. ROS were detected in EOMA cells, and images were acquired with a fluorescence microscope.

EOMA cells were seeded in 6-well plates for $22 \mathrm{~h}$ and subsequently incubated with various concentrations of tetrandrine, NAC $(20 \mathrm{mM})$ or pretreated with $20 \mathrm{mM}$ NAC for $1 \mathrm{~h}$ followed by $50 \mu \mathrm{M}$ tetrandrine. After incubation for $48 \mathrm{~h}$, EOMA cells were harvested, and cells were centrifuged (150 x g for $10 \mathrm{~min}$ ), supplemented with DCFH-DA solution $(1 \mu \mathrm{M})$ and incubated in the cell incubator at $37^{\circ} \mathrm{C}$ for $1 \mathrm{~h}$. The EOMA cells were centrifuged $(250 \mathrm{x}$ g for $10 \mathrm{~min})$ and washed with ice-cold PBS prior to flow cytometry (Beckman Coulter).

Tumor xenografts. Five-week-old male BALB/c nude mice were obtained from the Disease Prevention Centre of Hubei Province (Wuhan, China). The Experimental Animal Centre of Wuhan University approved the experimental protocols. The Huh7 cells were counted, and $2 \times 10^{7}$ cells were implanted in the right flank of each mouse. When the tumor volume reached $150-300 \mathrm{~mm}^{3}$, the mice were randomly distributed into control and treatment groups $(n=7)$ and gavaged. The control group received treatment with the vehicle, which consisted of $0.5 \%$ $(\mathrm{w} / \mathrm{v})$ methylcellulose and $0.1 \%(\mathrm{v} / \mathrm{v})$ Tween- 80 in sterile water. The treatment group was given tetrandrine at $50 \mathrm{mg} / \mathrm{kg}$ of body weight for 20 days, and the tumors were dissected and weighed.

Immunohistochemical analysis. CD31 panendothelial antigen (platelet/endothelial cell-adhesion molecule) was used for microvessel staining in frozen sections $(25,26)$. Frozen sections were then blocked with $5 \%$ goat serum and stained with rat anti-mCD31 antibody (1:50 dilution; BD BiosciencesPharmingen) at room temperature for $1 \mathrm{~h}$. Sections were then washed with PBS and incubated for $1 \mathrm{~h}$ with biotinylated polyclonal anti-rat IgG antibody. The sections were washed three times with PBS, reacted with the ABC peroxidase kit (Vector Laboratories) at room temperature for $45 \mathrm{~min}$, and washed twice with PBS prior to mounting for light microscopy and photography.

The number of vessels was counted in a blinded manner in 18 sections ( 2 sections per slide, 3 slides per tissue) of the tissue using a light microscope at x200 magnification. Five fields in each section were randomly selected, and the number of vessels in each field was averaged (27).

Statistical analysis. The results are expressed as the mean \pm SE. The Tukey-Kramer multiple comparisons test was used to determine statistical significance. A P-value of $<0.05$ was considered to be statistically significant.

\section{Results}

Tetrandrine inhibits the proliferation of EOMA cells. Tetrandrine, a compound isolated from a traditional Chinese medicine plant (Fig. 1A), has been found to be an antitumor 
A

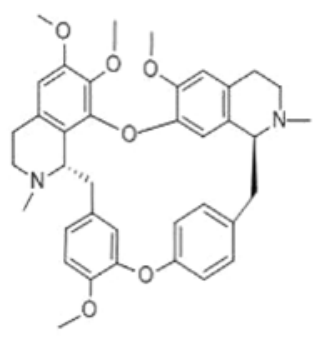

Chemical structure of Tetrandrine
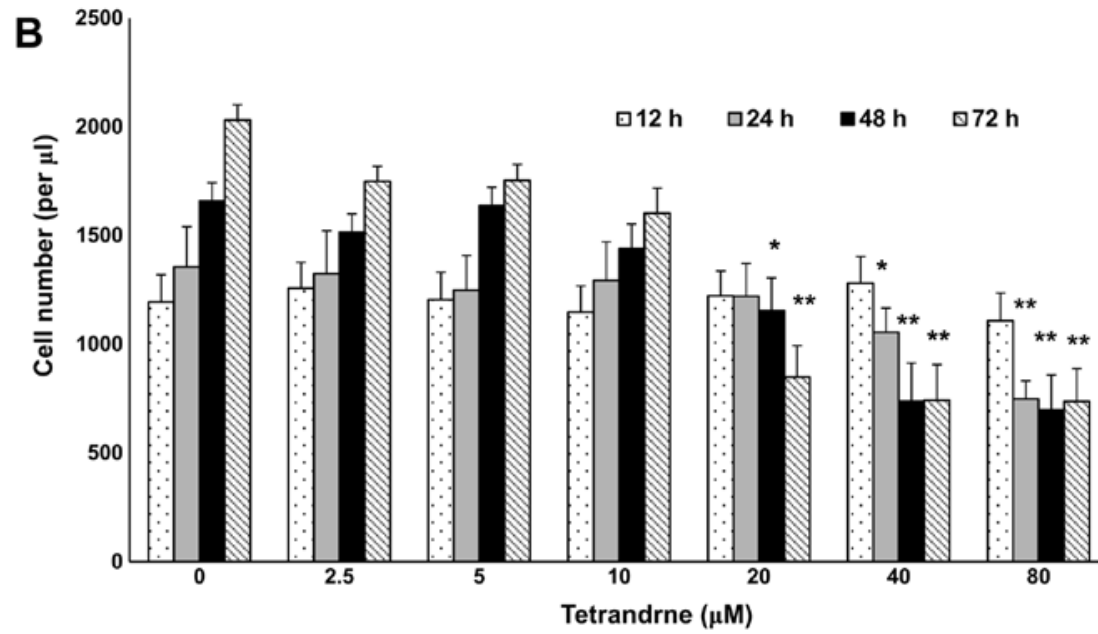

Tetrandrine $(\mu \mathrm{M})$

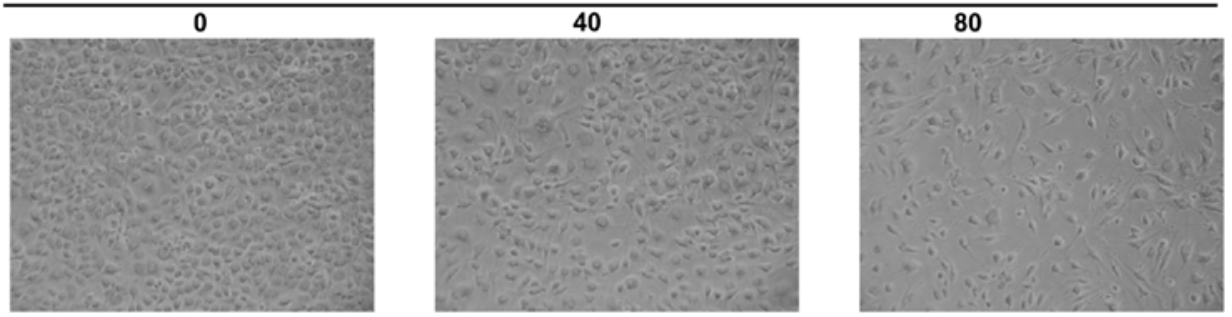

Figure 1. Tetrandrine (Tet) inhibits the proliferation of EOMA cells. The data represent an average of at least three independent experiments. (A) Chemical structure of tetrandrine $\left(\mathrm{C}_{38} \mathrm{H}_{42} \mathrm{~N}_{2} \mathrm{O}_{6}\right)$. (B) EOMA cells were treated with various concentrations of Tet for 12, 24, 48 and $72 \mathrm{~h}$, and cell numbers were counted in cell proliferation assay. The control cells were not treated with any drug. ${ }^{*} \mathrm{P}<0.05$ and ${ }^{* *} \mathrm{P}<0.01$ comparing with control, respectively. (C) Phase contrast microscopy of control EOMA cells and cells treated with 40 or $80 \mu \mathrm{M}$ tetrandrine for $48 \mathrm{~h}$.

agent (Fig. 1A) (10,28). After treatment with tetrandrine at indicated concentrations for 12, 24, 48 and 72 h, EOMA cells were analysed in proliferation assays. The proliferation of EOMA cells was inhibited, and the growth of EOMA cells was clearly suppressed after treatment with tetrandrine from 20 to $80 \mu \mathrm{M}$ for $48 \mathrm{~h}$ (Fig. $1 \mathrm{~B}$ and $\mathrm{C}$ ).

Tetrandrine affects the expression of G1/S cell cycle regulatory proteins in EOMA cells. Based on the above results, tetrandrine concentrations of 10,20,30,40 and $50 \mu \mathrm{M}$ and an incubation period of $48 \mathrm{~h}$ were selected as conditions for cell cycle analysis by flow cytometry. As shown in Fig. 2A, the proportion of EOMA cells in $\mathrm{S}$ phase was decreased from $20.8 \%$ in control cells to $19.0,16.0,16.2,14.2$ and $13.9 \%$ in cells treated with tetrandrine for $48 \mathrm{~h}$ at concentrations of $10,20,30,40$ and $50 \mu \mathrm{M}$, respectively, while the proportion of cells in $\mathrm{G} 1 / \mathrm{G} 0$ was increased from 60.9 to $63.7,67.1,62.7,63.9$ and $67.5 \%$ under the same treatments. Thus, these results indicated that tetrandrine induced G1/S cell cycle arrest in EOMA cells in a dose-dependent manner.

To further investigate the observed G1/S cell cycle arrest, cell cycle regulation proteins, including cyclin D1, cyclin D2, cyclin D3, cyclin E1, cyclin E2, CDK2, CDK4 and CDK6 were determined by western blotting. The expression levels of cyclin D1, cyclin D2, cyclin E1, cyclin E2, CDK2 CDK4 and CDK6 were decreased in tetrandrine-treated cells compared to the control cells, but the expression of cyclin D3 was not affected by tetrandrine treatment (Fig. 2B). In addition, the expression levels of the proteins, including GSK-3 $\beta$, p53 and $\mathrm{p} 27$, which were the inhibitors for the cell cycle regulators, were upregulated with tetrandrine treatment (Fig. 2C).

Tetrandrine upregulates the ROS level in EOMA cells. Since tetrandrine has been reported as an anticancer agent both in vitro and in vivo by inducing apoptosis through the formation of ROS (29), which can serve as important second messengers to regulate a variety of downstream signalling pathways (30-32), we determined the effect of tetrandrine on intracellular ROS levels in EOMA cells using DCFH-DA fluorescence. As shown in Fig. 3A, the number of fluorescent sites, representing the level of ROS, was higher in tetrandrinetreated cells than in the control cells. Flow cytometry results also showed that the ROS level was increased by treatments with 30 and $50 \mu \mathrm{M}$ of tetrandrine as compared to the control, up to 2-fold in the $50 \mu \mathrm{M}$ tetrandrine-treated cells (Fig. 3B). These results demonstrate that ROS was produced in EOMA cells in response to tetrandrine.

$N A C$ inhibits $G 1 / S$ cell cycle arrest in tetrandrine-treated EOMA cells. To further investigate the role of ROS in the upregulation of tetrandrine-treated EOMA cells, the ROS inhibitor was used. ROS accumulation was observed in EOMA cells after $48 \mathrm{~h}$ of tetrandrine treatment, but was markedly abrogated when cells were pretreated with the ROS scavenger NAC, demonstrating an effective elimination of tetrandrine-induced ROS production by NAC (Fig. 4A). Also in the cell cycle analysis, pretreatment with NAC inhibited 


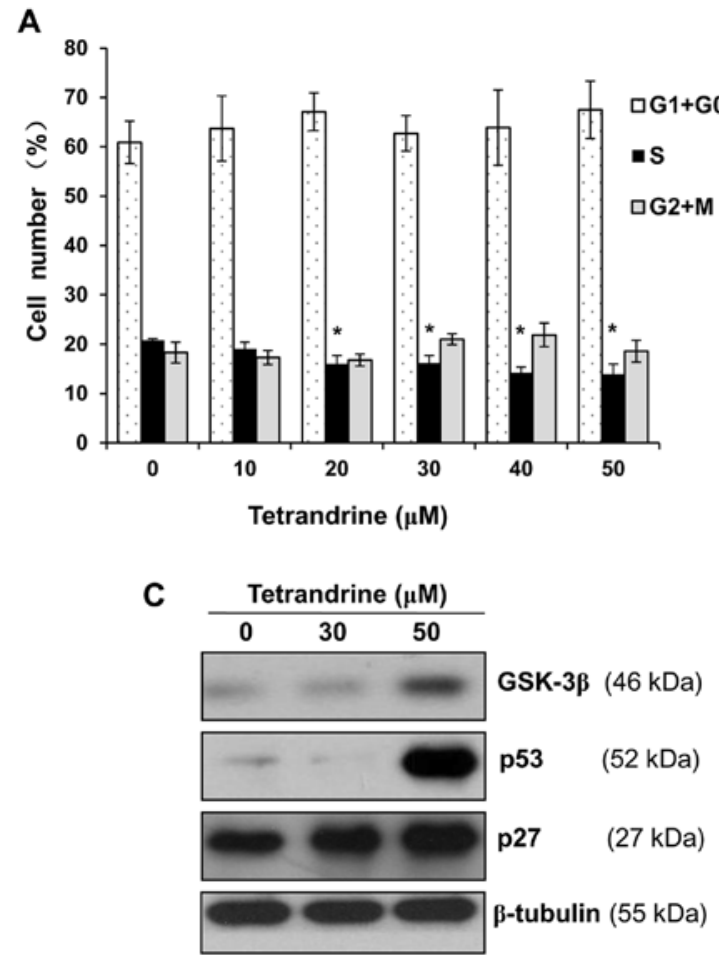

B

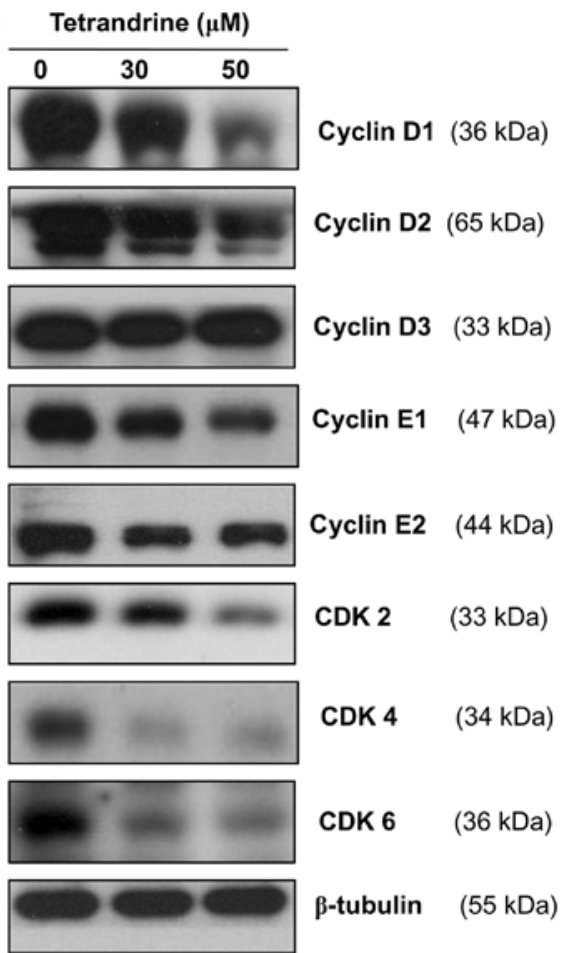

Figure 2. Tetrandrine affects the expression of G1/S cell cycle regulatory proteins in EOMA cells. The data represent an average of at least three independent experiments. (A) Statistical analysis of the percentage of cells in G0/G1 and S phase of the cell cycle in the EOMA cells. RNase and PI were added to the cell suspension after EOMA cells were treated with various tetrandrine concentrations for $48 \mathrm{~h}$, and flow cytometry was used to detect the proportions of cells at different phases of the cell cycle. The control group was not treated with any drug. " $\mathrm{P}<0.05$ comparing with control. (B and C) EOMA cells were treated with 30 or $50 \mu \mathrm{M}$ tetrandrine for $48 \mathrm{~h}$, and western blot assays were performed to detect the levels of cell cycle-related proteins. $\beta$-tubulin was used as the reference. The control group was not treated with any drug.

A

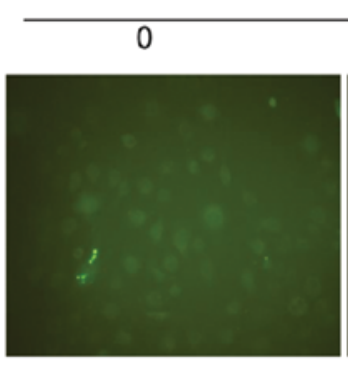

B

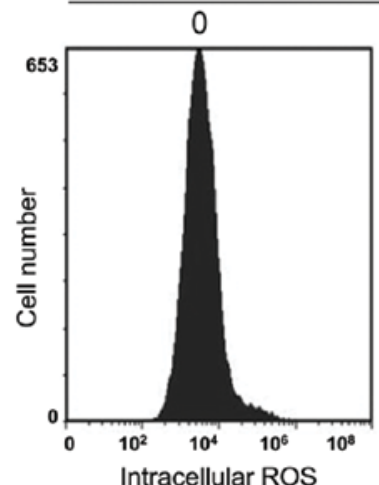

Tetrandrine $(\mu \mathrm{M})$
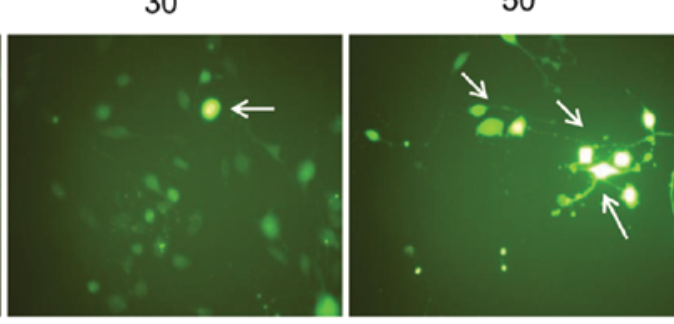

Tetrandrine $(\mu \mathrm{M})$
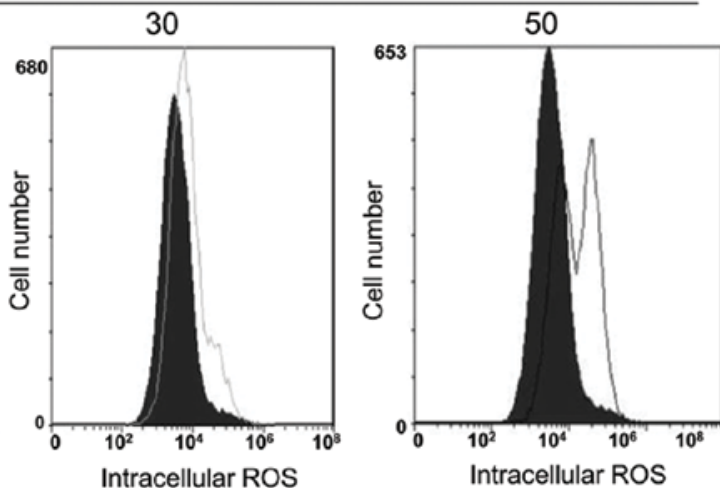
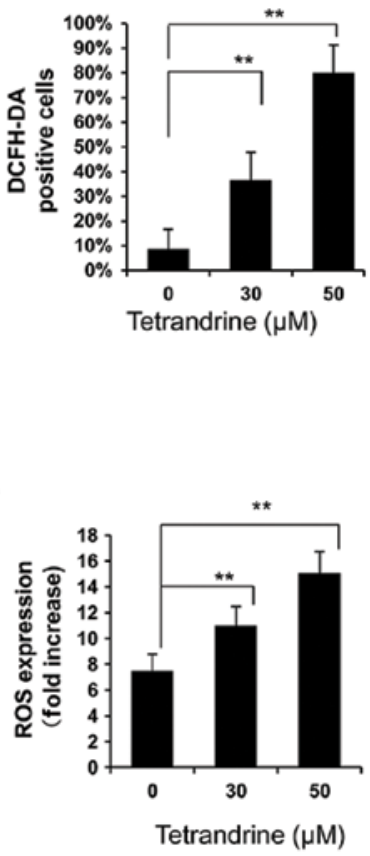

Figure 3. Tetrandrine upregulates the level of reactive oxygen species (ROS) in EOMA cells. The data represent an average of at least three independent experiments. (A) DCFH-DA was added to stain the cells after EOMA cells were treated with 30 and $50 \mu \mathrm{M}$ tetrandrine for $48 \mathrm{~h}$. Fluorescence microscopy was used to observe the ROS in the cells and statistical analysis of ROS fluorescence is shown. The fluorescent sites are indicated by arrows. The control group was not treated with any drug (magnification, $\mathrm{x} 200$ ). ${ }^{* *} \mathrm{P}<0.01$. (B) DCFH-DA was added to stain the EOMA cells after treatment with 30 or $50 \mu \mathrm{M}$ tetrandrine for $48 \mathrm{~h}$. Flow cytometry was applied to analyse the level of ROS in cells and statistical analysis of ROS fluorescence after treatment with 30 or $50 \mu \mathrm{M}$ tetrandrine is shown. The control cells were not treated with any drug. 

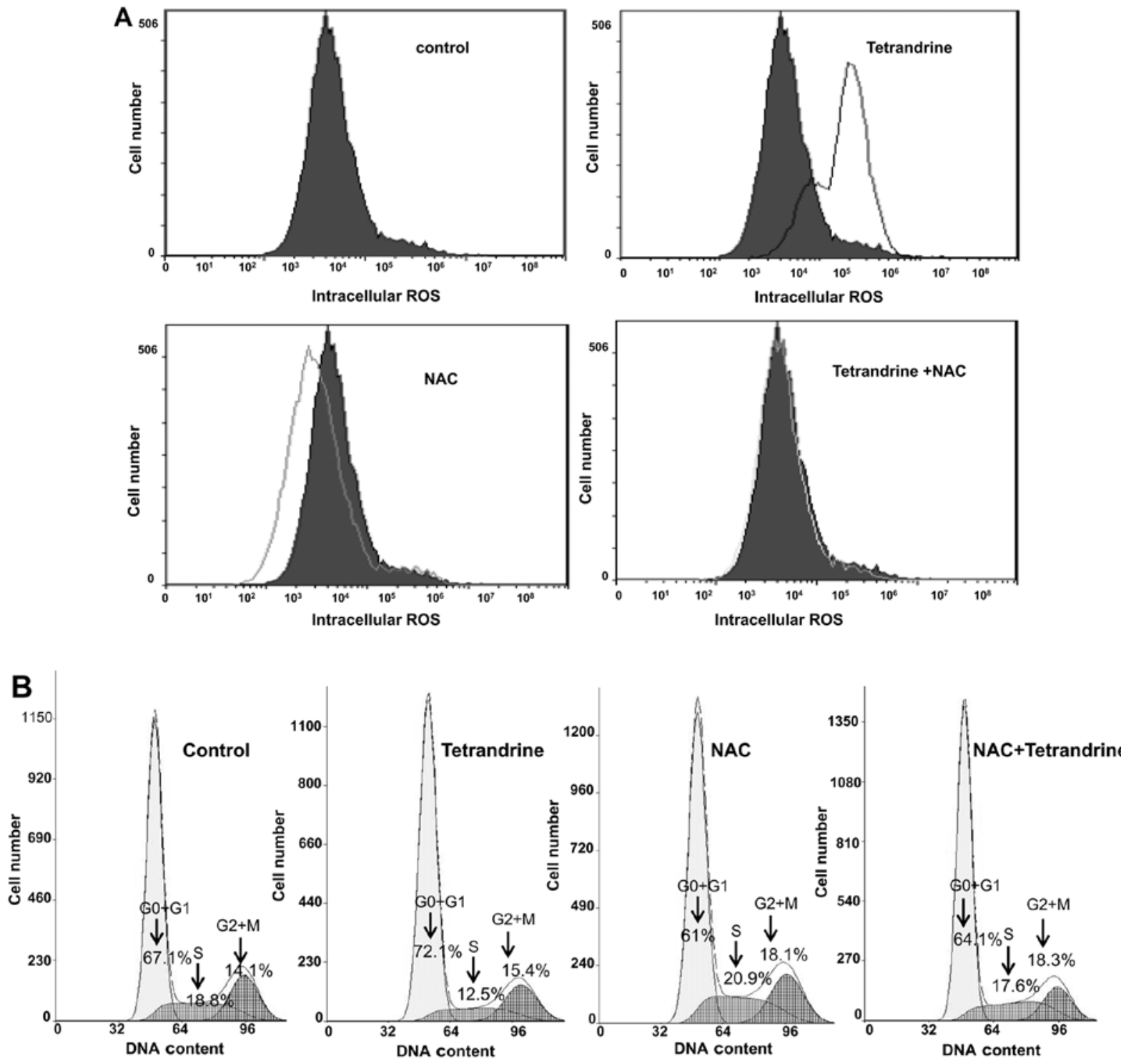

C

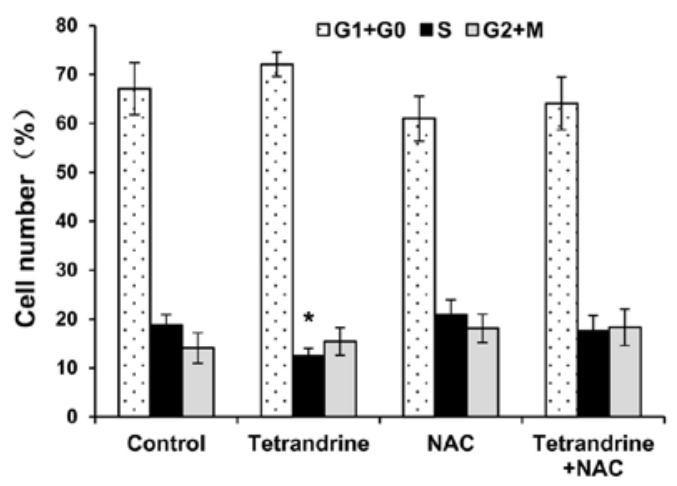

D

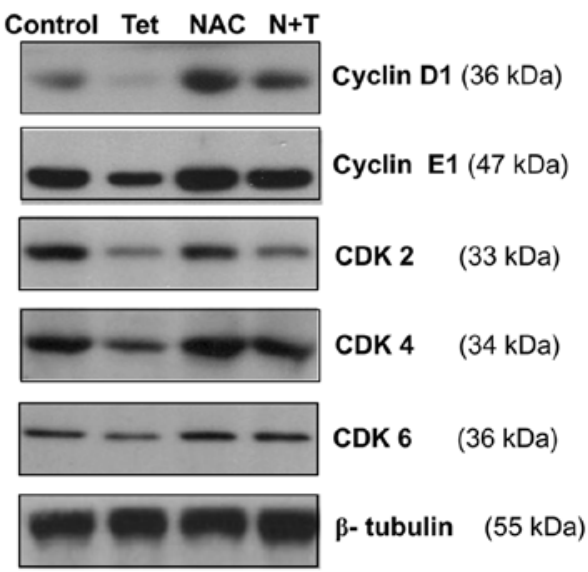

Figure 4. NAC rescues G1/S cell cycle arrest in tetrandrine-treated EOMA cells. (A) Cells were treated with $20 \mathrm{mM} \mathrm{NAC}$ for $1 \mathrm{~h}$ prior to the addition of $50 \mu \mathrm{M}$ tetrandrine. DCFH-DA was added to stain the cells to detect the level of ROS. The control cells were not treated with any drug. (B) Cells were treated with $20 \mathrm{mM} \mathrm{NAC}$ for $1 \mathrm{~h}$ prior to the addition of $50 \mu \mathrm{M}$ tetrandrine. RNase and PI were added to analyse the different phases of the cell cycle. The control cells were not treated with any drug. (C) Statistical analysis of the percentage of cells in G0/G1 and S phase of the cell cycle in the EOMA cells. "P<0.05 comparing with control. (D) Cells were treated with $20 \mathrm{mM} \mathrm{NAC}$ for $1 \mathrm{~h}$ prior to the addition of $50 \mu \mathrm{M}$ tetrandrine, and western blot assays were used to detect the levels of cyclin D1, cyclin E1, CDK2, CDK4 and CDK6. $\beta$-tubulin was used as the reference. The control cells were not treated with any drug.

the G1/S cell cycle arrest in tetrandrine-treated EOMA cells (Fig. 4B and C).
To determine the association between cell cycle arrest and the increased ROS in EOMA cells, the expression levels of 
A

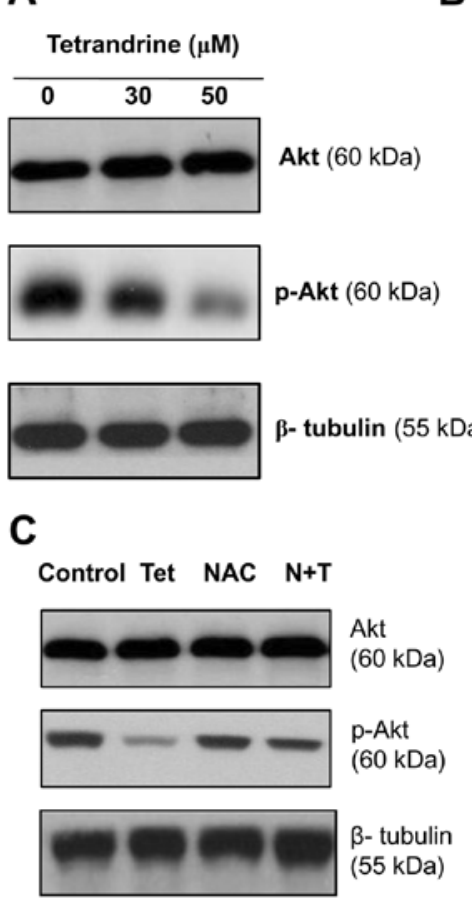

B
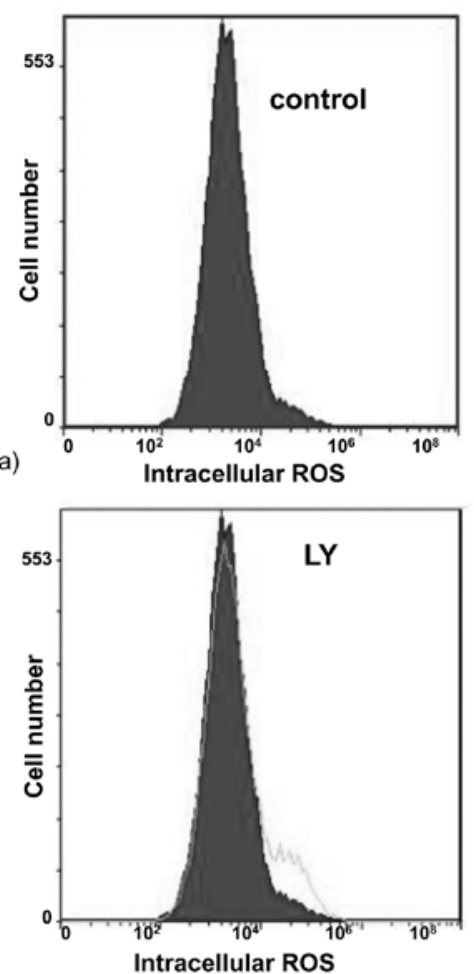

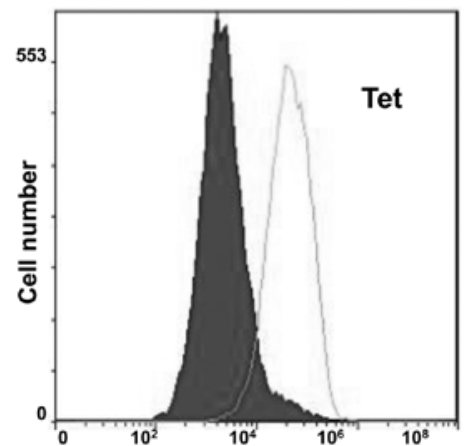

Intracellular ROS

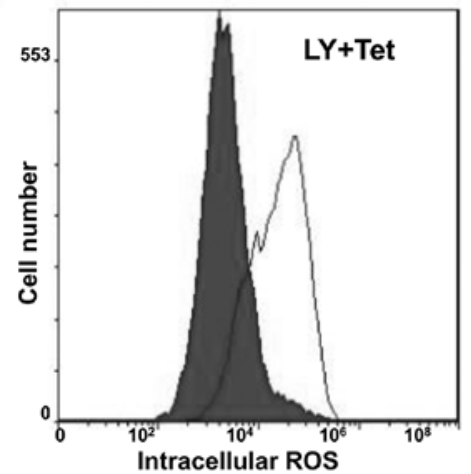

Figure 5. Tetrandrine (Tet) inhibits the PI3K-Akt signal pathway, and NAC rescues the inhibition of Akt in tetrandrine-treated EOMA cells. (A) EOMA cells were treated with 30 and $50 \mu \mathrm{M}$ tetrandrine for $48 \mathrm{~h}$, and a western blot assay was applied to detect the level of total Akt and p-Akt (ser473) in EOMA cells. (B) Cells were treated with $1.6 \mu \mathrm{M} \mathrm{LY}$ for $1 \mathrm{~h}$ prior to the addition of $50 \mu \mathrm{M}$ tetrandrine, and flow cytometry was used to analyse the level of ROS in cells after DCFH-DA was added to stain the cells. (C) Cells were treated with $20 \mathrm{mM}$ NAC for $1 \mathrm{~h}$ prior to the addition of $50 \mu \mathrm{M}$ tetrandrine, and a western blot assay was applied to detect the level of total Akt and p-Akt (ser473) in EOMA cells. $\beta$-tubulin was used as a reference.

cyclins and their upstream proteins were analysed by western blotting. The downregulation of cyclin D1, cyclin E1, CDK2, CDK4 and CDK6 in EOMA cells was inhibited by pretreatment with $20 \mathrm{mM}$ of NAC for $1 \mathrm{~h}$ prior to treatment with $50 \mu \mathrm{M}$ of tetrandrine for $48 \mathrm{~h}$ (Fig. 4D).

A decreased phospho-Akt protein level after tetrandrine treatment was reversible with the removal of the intracellular $R O S$ by NAC. It is known that ROS generation is related with the $\mathrm{PI} 3 \mathrm{~K}-\mathrm{Akt}$ signalling pathway. Akt is a critical kinase that regulates a variety of biological processes, including cell survival, proliferation, autophagy and apoptosis (33-36). To identify if Akt was involved in tetrandrine treated EOMA cells, western blots were performed as previously described. As shown in Fig. 5A, the level of phosphorylated Akt was decreased after tetrandrine treatment although the total Akt protein level remained unchanged. Then, to determine whether tetrandrine induced ROS upregulation by decreasing Akt activity, the EOMA cells were treated with tetrandrine in combination with LY294002 (a PI3K/Akt inhibitor) for $48 \mathrm{~h}$ and the ROS level was determined by flow cytometry. The results indicate that LY294002 did not inhibit tetrandrine-induced increase of ROS in EOMA cells (Fig. 5B).

Next, to determine whether the phosphorylation of Akt was regulated by the increased levels of ROS, the EOMA cells were pretreated with $20 \mathrm{mM}$ of NAC for $1 \mathrm{~h}$ followed by treatment with $50 \mu \mathrm{M}$ of tetrandrine for $48 \mathrm{~h}$, and both total Akt and phosphorylated Akt were analysed by western blotting. As shown in Fig. 5C, the tetrandrine-induced decrease of Akt phosphorylation was blocked by NAC pretreatment. Taken together, these data suggest that ROS acted upstream of the Akt pathway to regulate the cell cycle.

Overexpression of Akt decreased tetrandrine-induced G1/S arrest. To further establish that Akt was involved in tetrandrine-induced G1/S arrest, empty vector (PUSE) or PUSE-CA-Akt was transfected into EOMA cells. After tetrandrine treatment, the cell number of EOMA cells with Akt overexpression was nearly twice that of the control cells (Fig. 6A). It indicated that Akt overexpression could promote cell cycle transition and then had an effect on cell proliferation. Next, even with tetrandrine treatment, it showed that the proportion in $\mathrm{S}$ phase of EOMA cells with Akt overexpression had a significantly increase to $41.2 \%$, compared with the control cells with $28.9 \%$ in $\mathrm{S}$ phase (Fig. 6B and C). Finally, the western blot analysis also checked the expression levels of cyclin D1, cyclin E1, CDK2, CDK4 and CDK6, which were important for G1/S arrest. As shown in Fig. 6D, when EOMA cells were transfected with the control vector, and the cells were treated with tetrandrine, cyclin D1, cyclin E1, CDK2, CDK4 and CDK6 were significantly downregulated. However, when the EOMA cells were transfected with Akt overexpressing vector, and treated with tetrandrine, the protein levels did not change much comparing with the control cells. This also suggested that tetrandrine-induced G1/S cell cycle arrest was rescued because of the overexpression of Akt. Collectively, considering the above, we concluded that the 

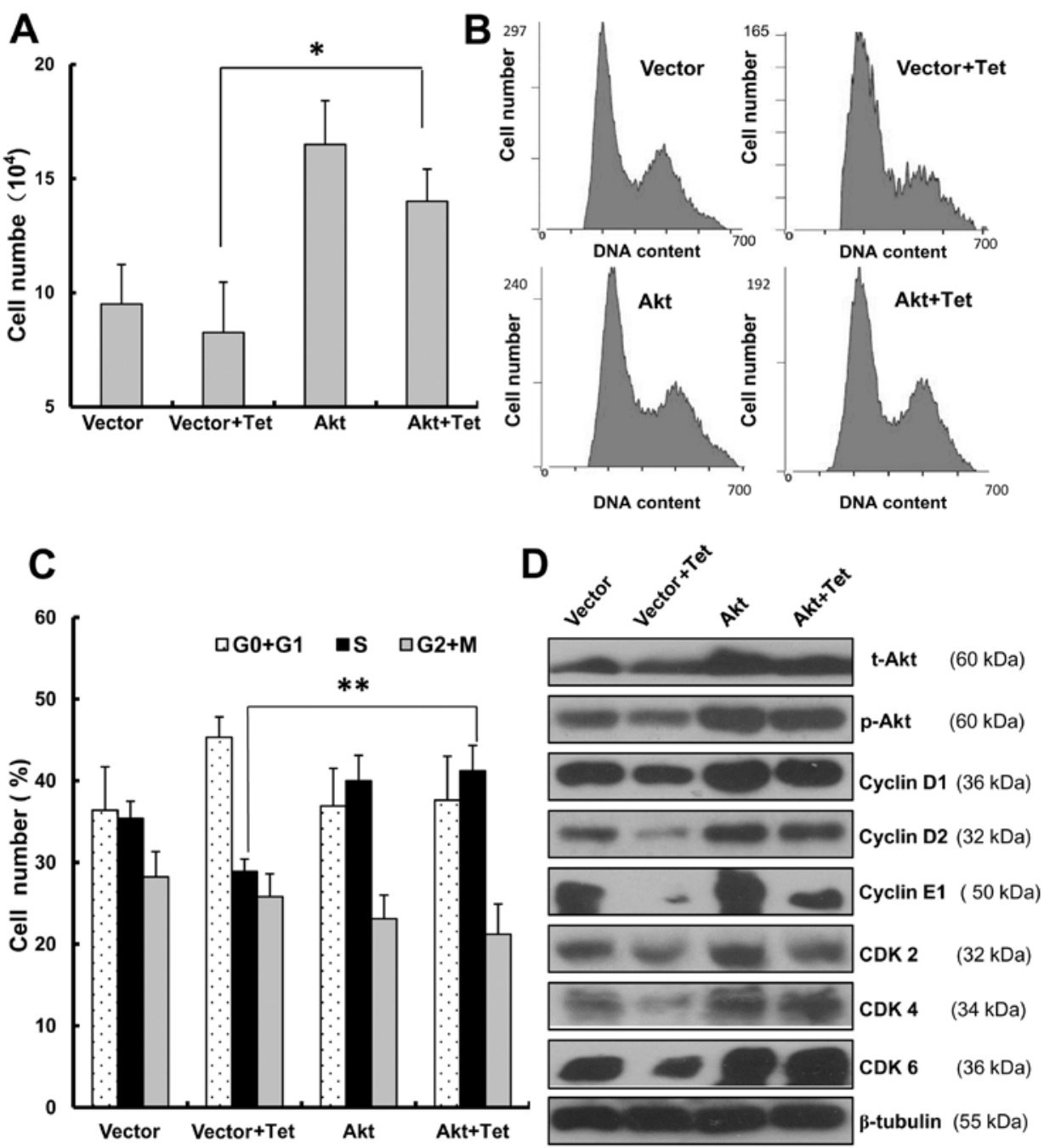

Figure 6. Overexpression of Akt decreased tetrandrine-induced G1/S arrest. The data represent an average of at least three independent experiments. EOMA cells $\left(5 \times 10^{4}\right)$ were placed into 6-well plates. After $23 \mathrm{~h}$, cells were transfected with PUSE or PUSE-CA-Akt. One day later, cells were incubated with or without tetrandrine at a concentration of $30 \mu \mathrm{M}$ for two days. Cell numbers were counted in cell proliferation assay. After the treatment with tetrandrine, the proliferation rate of Akt overexpressed EOMA cells was higher than the cells that was transfected with only control vector. ${ }^{*} \mathrm{P}<0.05$ comparing with control (B) Cells were treated with $20 \mathrm{mM} \mathrm{NAC}$ for $1 \mathrm{~h}$ prior to the addition of $50 \mu \mathrm{M}$ tetrandrine. RNase and PI were added to analyse the different phases of the cell cycle. (C) Statistical analysis of the percentage of cells in G0/G1 and S phase of the cell cycle in the EOMA cells. Overexpression of Akt caused an increase of cell population percentage in $\mathrm{S}$ phase from 28.9 to $41.2 \%$ comparing with the control cells. ${ }^{* *} \mathrm{P}<0.01$ comparing with control. (D) Western blot assays were used to detect the levels of cyclin D1, cyclin E1, CDK2, CDK4 and CDK6. $\beta$-tubulin was used as the reference. Tetrandrine-induced suppression of cyclin D1, cyclin D2, cyclin E1, CDK2 and CDK4 (lane 2) were completely rescued (lane 4) by overexpression of Akt.

overexpression of Akt decreases tetrandrine-induced G1/S arrest.

Tetrandrine inhibits angiogenesis in liver cancer in vivo. Since the regulation of the endothelial cell cycle is critical to the function of the vascular system (14), it suggests that tetrandrine may be an effective angiogenesis inhibitor in cancer therapy. To evaluate the antitumor effect of tetrandrine in vivo, we investigated whether tetrandrine could inhibit tumor vessel growth in nude mice. As previously described, the mice bearing Huh7 tumor xenografts were gavaged with tetrandrine $(50 \mathrm{mg} /$ $\mathrm{kg}$ of body weight) or vehicle every other day, and the amount of vessels inside the tumor was observed in tumor sections after 20 days of treatment (9). Then we found the tumor vascular growth was visually inhibited by tetrandrine, and the data confirmed its anti-angiogenic activity (Fig. 7A). Vascular density was also measured in anti-CD31 stained vasculatures from both control tumor mice and tetrandrine-treated tumor mice. The results of immunohistochemical analysis showed a significant decrease of microvessel density in tetrandrinetreated group (Fig. 7B), further demonstrating the inhibition of liver cancer vascular growth in nude mice bearing liver cancer xenografts. Consistent with the reduction of tumor vascular growth by tetrandrine treatment in vivo, the average tumor weight in the animals treated with vehicle, tetrandrine therapy was 1.11 and $0.53 \mathrm{~g}(\mathrm{P}<0.01)$, respectively (Fig. 7C). Therefore, tetrandrine also has antitumor effect and one of the important reasons is its anti-angiogenic function.

\section{Discussion}

In the present study, tetrandrine decreased cyclin D, cyclin E and CDKs expression, and increased the expression levels of the inhibitors of G1/S cell cycle arrest in endothelial cells, 
A

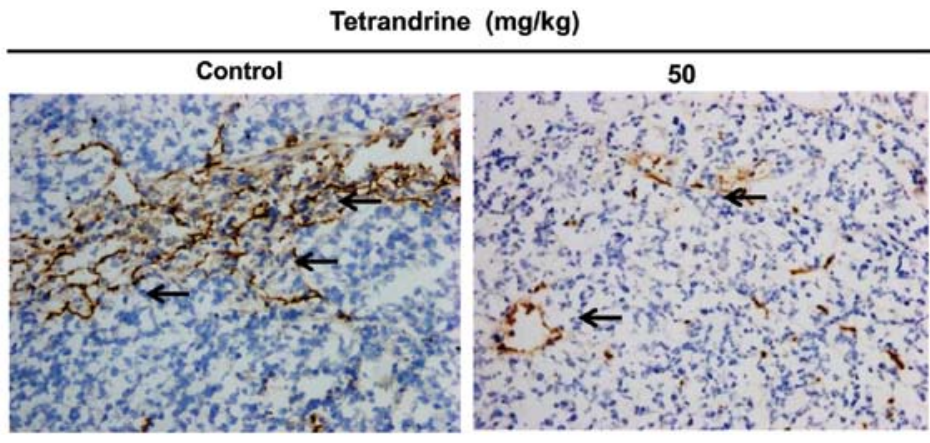

B

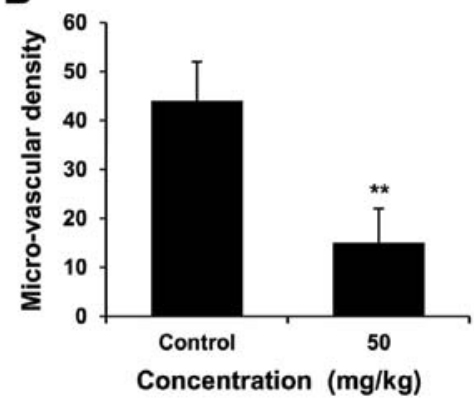

C

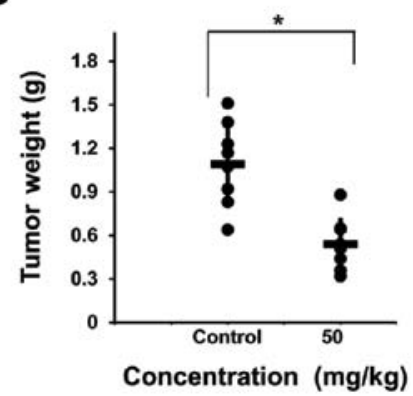

D

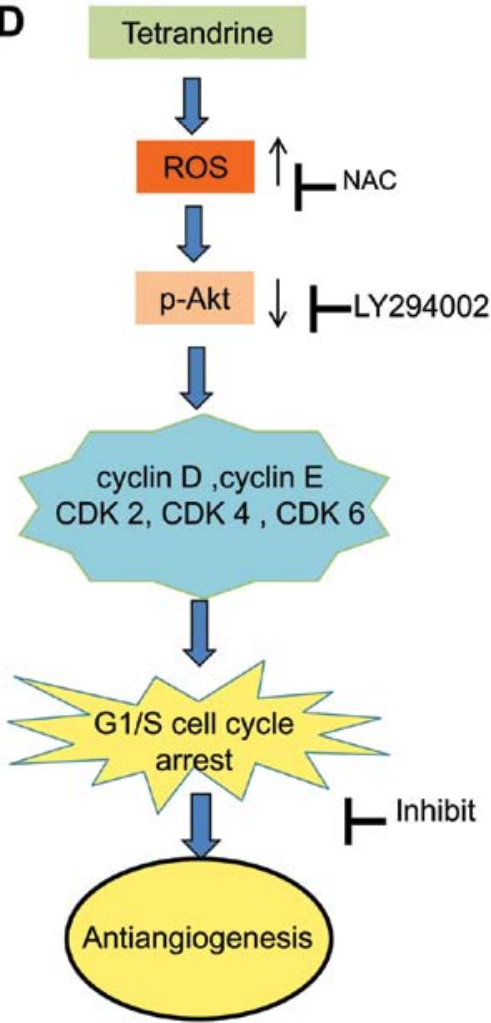

Figure 7. Tetrandrine inhibits angiogenesis in liver cancer in vivo. (A) Tumors were removed from the nude mice after treatment with 50 mg/kg tetrandrine, and subsequently marked with CD31. Slices of the frozen tumors were subjected to immunohistochemistry to detect the vascular density in the tissues, and haematoxylin was added to detect the cell nuclei. The arrows indicated CD31 positive expressions. An inverted microscope was used to observe the slides (magnification, x200). (B) Statistical analysis of the micro-vessel densities in different tissues. The control group was treated with $0.1 \%$ carboxymethyl cellulose sodium solution. ${ }^{* *} \mathrm{P}<0.01$ comparing with control. (C) Tumor tissues were weighed from Huh7 xenografts treated with tetrandrine. ${ }^{*}<0.05$ comparing with control. Values represent the means $\pm \mathrm{SD}$. (N=7). (D) Putative model for the signaling pathway that mediates tetrandrine-induced antiangiogenesis.

resulting in $\mathrm{G} 1 / \mathrm{S}$ arrest and anti-angiogenesis. A previous study also reported that tetrandrine combined with cisplatin enhances cytotoxicity and could induce G1 arrest and apoptosis in ovarian cancer cells (37). In human colon carcinoma HT-29 cells, tetrandrine-induced Akt dephosphorylation was observed, and GSK-3 $\beta$ was able to induce subsequent degradation of cyclin D1 (38).

Usually physiological stimuli are capable of inducing Akt kinase activity through PI3 kinase, and ROS generation is dependent on the PI3K-Akt signalling pathway $(33,39,40)$. To determine the relationship between ROS generation and phosphorylated Akt inhibition in tetrandrine-induced endothelial cell cycle arrest, NAC (a ROS inhibitor) and LY294002 (a PI3K/Akt inhibitor) were used to treat EOMA cells. Phosphorylated Akt levels were decreased after tetrandrine treatment, and this decrease was prevented by NAC. Then it showed that tetrandrine-induced ROS generation was not inhibited by LY294002 in EOMA cells. This result suggested that ROS acts upstream of the PI3K/Akt signalling pathway, while Akt did not have an effect on the ROS accumulation.

Further experiments demonstrated that when EOMA cells were overexpressed with Akt, tetrandrine-induced G1/S arrest was inhibited indicating that tetrandrine can inhibit cell proliferation through PI3K/Akt pathway. The PI3K/Akt/mTOR pathway has an important role in cell metabolism, growth, migration, survival and angiogenesis. Drug development aimed at this pathway has been performed and is a frequent occurrence in human cancer (40-42).

The data revealed that tetrandrine inhibited tumor angiogenesis in vivo in Huh7 tumor-implanted nude mice. Because CD31 is an endothelial marker, anti-CD31 staining was used to assess the vascular density $(27,43)$. The result confirmed that the vascular density in the tumors in control mice was higher than that in the tumors treated with tetrandrine. Additionally, the tumors weight in the mice that were treated with tetrandrine was lower than the mice that received vehicle only.

In summary, tetrandrine induces $\mathrm{G} 1 / \mathrm{S}$ cell cycle arrest in EOMA cells by activating ROS and repressing Akt phosphorylation, and ROS appears to be an upstream regulator of Akt. Thus, tetrandrine possesses anti-angiogenic activity by inhibiting EOMA cells proliferation via ROS/Akt pathway (Fig. 7D). In vivo experiments also demonstrated that tetrandrine reduces the vascular density and inhibits tumor growth. Therefore, our data suggest that tetrandrine is a candidate for development as an anti-angiogenic agent.

\section{Acknowledgements}

Authors wish to thank Center for Medical Research in Wuhan University for the help with the flow cytometric analysis. The present study was supported by the National Program on Key Basic Research Project (973 Program, no. 2010CB529804), the National Natural Science Foundation of China (grant nos. 
30971456, 31400155, 81472550, 81274048 and 81273540), and by the Innovation Seed Fund of Wuhan University School of Medicine.

\section{References}

1. Gerald D, Chintharlapalli S, Augustin HG and Benjamin LE: Angiopoietin-2: an attractive target for improved antiangiogenic tumor therapy. Cancer Res 73: 1649-1657, 2013.

2. Turley RS, Fontanella AN, Padussis JC, et al: Bevacizumabinduced alterations in vascular permeability and drug delivery: a novel approach to augment regional chemotherapy for in-transit melanoma. Clin Cancer Res 18: 3328-3339, 2012.

3. Peng F, Xu Z, Wang J, et al: Recombinant human endostatin normalizes tumor vasculature and enhances radiation response in xenografted human nasopharyngeal carcinoma models. PLoS One 7: e34646, 2012.

4. Sagar SM, Yance D and Wong RK: Natural health products that inhibit angiogenesis: a potential source for investigational new agents to treat cancer - Part 2. Curr Oncol 13: 99-107, 2006.

5. Li SY, Ling LH, Teh BS, Seow WK and Thong YH: Antiinflammatory and immunosuppressive properties of the bis-benzylisoquinolines: in vitro comparisons of tetrandrine and berbamine. Int J Immunopharmacol 11: 395-401, 1989.

6. Chen Y, Chen JC and Tseng SH: Tetrandrine suppresses tumor growth and angiogenesis of gliomas in rats. Int J Cancer 124 2260-2269, 2009.

7. Lai JH: Immunomodulatory effects and mechanisms of plant alkaloid tetrandrine in autoimmune diseases. Acta Pharmacol Sin 23: 1093-1101, 2002.

8. Wang G, Lemos JR and Iadecola C: Herbal alkaloid tetrandrine: fron an ion channel blocker to inhibitor of tumor proliferation. Trends Pharmacol Sci 25: 120-123, 2004.

9. Liu C, Gong K, Mao X and Li W: Tetrandrine induces apoptosis by activating reactive oxygen species and repressing Akt activity in human hepatocellular carcinoma. Int J Cancer 129: 1519-1531, 2011.

10. Lee JH, Kang GH, Kim KC, et al: Tetrandrine-induced cell cycle arrest and apoptosis in A549 human lung carcinoma cells. Int J Oncol 21: 1239-1244, 2002.

11. Li X, Su B, Liu R, Wu D and He D: Tetrandrine induces apoptosis and triggers caspase cascade in human bladder cancer cells. J Surg Res 166: e45-51, 2011.

12. Wu JM, Chen Y, Chen JC, Lin TY and Tseng SH: Tetrandrine induces apoptosis and growth suppression of colon cancer cells in mice. Cancer Lett 287: 187-195, 2010.

13. Gong K, Chen C, Zhan Y, Chen Y, Huang Z and Li W: Autophagy-related gene 7 (ATG7) and reactive oxygen species/ extracellular signal-regulated kinase regulate tetrandrineinduced autophagy in human hepatocellular carcinoma. J Bio Chem 287: 35576-35588, 2012.

14. Yu J, Tian S, Metheny-Barlow L, et al: Modulation of endothelial cell growth arrest and apoptosis by vascular endothelial growth inhibitor. Circ Res 89: 1161-1167, 2001.

15. Kuo PL and Lin CC: Tetrandrine-induced cell cycle arrest and apoptosis in Hep G2 cells. Life Sci 73: 243-252, 2003.

16. Kobayashi S, Inaba K, Kimura I and Kimura M: Inhibitory effects of tetrandrine on angiogenesis in adjuvant-induced chronic inflammation and tube formation of vascular endothelial cells. Biol Pharm Bull 21: 346-349, 1998.

17. Gao JL, Ji X, He TC, et al: Tetrandrine suppresses cancer angiogenesis and metastasis in 4T1 tumor bearing mice. Evid Based Complement Alternat Med 2013: 265061, 2013.

18. Felbor U, Dreier L, Bryant RA, Ploegh HL, Olsen BR and Mothes W: Secreted cathepsin L generates endostatin from collagen XVIII. EMBO J 19: 1187-1194, 2000.

19. Wen W, Moses MA, Wiederschain D, Arbiser JL and Folkman J: The generation of endostatin is mediated by elastase. Cancer Res 59: 6052-6056, 1999.

20. Gordillo GM, Onat D, Stockinger M, et al: A key angiogenic role of monocyte chemoattractant protein-1 in hemangioendothelioma proliferation. Am J Physiol Cell Physiol 287: C866-C873, 2004.

21. Obeso J, Weber J and Auerbach R: A hemangioendotheliomaderived cell line: its use as a model for the study of endothelial cell biology. Lab Invest 63: 259-269, 1990.
22. Lannutti BJ, Gately ST, Quevedo ME, Soff GA and Paller AS: Human angiostatin inhibits murine hemangioendothelioma tumor growth in vivo. Cancer Res 57: 5277-5280, 1997.

23. O'Reilly MS, Brem H and Folkman J: Treatment of murine hemangioendotheliomas with the angiogenesis inhibitor AGM-1470. J Pediatr Surg 30: 325-330, 1995.

24. O'Reilly MS, Boehm T, Shing Y, et al: Endostatin: an endogenous inhibitor of angiogenesis and tumor growth. Cell 88: 277-285, 1997.

25. Koukourakis MI, Giatromanolaki A, Thorpe PE, et al: Vascular endothelial growth factor/KDR activated microvessel density versus CD31 standard microvessel density in non-small cell lung cancer. Cancer Res 60: 3088-3095, 2000.

26. Qin L, Zhao D, Liu X, et al: Down syndrome candidate region 1 isoform 1 mediates angiogenesis through the calcineurin-NFAT pathway. Mol Cancer Res 4: 811-820, 2006.

27. Davani S, Marandin A, Mersin N, et al: Mesenchymal progenitor cells differentiate into an endothelial phenotype, enhance vascular density, and improve heart function in a rat cellular cardiomyoplasty model. Circulation 108 (Suppl 1): II253-II258, 2003.

28. Li X, Lu X, Xu H, et al: Paclitaxel/tetrandrine coloaded nanoparticles effectively promote the apoptosis of gastric cancer cells based on 'oxidation therapy'. Mol Pharm 9: 222-229, 2012.

29. Heinzelmann S and Bauer G: Multiple protective functions of catalase against intercellular apoptosis-inducing ROS signaling of human tumor cells. Biol Chem 391: 675-693, 2010.

30. Fatehi-Hassanabad Z, Chan CB and Furman BL: Reactive oxygen species and endothelial function in diabetes. Eur J Pharmacol 636: 8-17, 2010

31. Ferreira AK, de-Sa-Junior PL, Pasqualoto KF, et al: Cytotoxic effects of dillapiole on MDA-MB-231 cells involve the induction of apoptosis through the mitochondrial pathway by inducing an oxidative stress while altering the cytoskeleton network. Biochimie 99: 195-207, 2013.

32. Gong K, Xie J, Yi H and Li W: CS055 (Chidamide/HBI-8000), a novel histone deacetylase inhibitor, induces G1 arrest, ROS-dependent apoptosis and differentiation in human leukaemia cells. Biochem J 443: 735-746, 2012.

33. Sheppard K, Kinross KM, Solomon B, Pearson RB and Phillips WA: Targeting PI3 kinase/AKT/mTOR signaling in cancer. Crit Rev Oncog 17: 69-95, 2012.

34. Janku F, McConkey DJ, Hong DS and Kurzrock R: Autophagy as a target for anticancer therapy. Nat Rev Clin Oncol 8: 528-539, 2011.

35. Song MS, Salmena L and Pandolfi PP: The functions and regulation of the PTEN tumour suppressor. Nat Rev Mol Cell Biol 13: 283-296, 2012.

36. Liu P, Begley M, Michowski W, et al: Cell-cycle-regulated activation of Akt kinase by phosphorylation at its carboxyl terminus. Nature 508: 541-545, 2014.

37. Zhang Y, Wang C, Wang H, Wang K, Du Y and Zhang J: Combination of Tetrandrine with cisplatin enhances cytotoxicity through growth suppression and apoptosis in ovarian cancer in vitro and in vivo. Cancer Lett 304: 21-32, 2011.

38. McCubrey JA, Basecke J, Cervello M, Martelli AM and Franklin RA: GSK-3beta is a critical mediator of tetrandrine induced cell cycle arrest and cytotoxicity. Cancer Biol Ther 7: 1079,2008

39. Lee HG, Lee YJ and Yang JH: Perfluorooctane sulfonate induces apoptosis of cerebellar granule cells via a ROS-dependent protein kinase C signaling pathway. Neurotoxicology 33: 314-320, 2012.

40. Lu CY, Yang YC, Li CC, Liu KL, Lii CK and Chen HW: Andrographolide inhibits TNFalpha-induced ICAM-1 expression via suppression of NADPH oxidase activation and induction of HO-1 and GCLM expression through the PI3K/Akt/Nrf2 and PI3K/Akt/AP-1 pathways in human endothelial cells. Biochem Pharmacol 91: 40-50, 2014.

41. Muniz-Feliciano L, Van Grol J, Portillo JA, et al: Toxoplasma gondii-induced activation of EGFR prevents autophagy proteinmediated killing of the parasite. PLoS Pathog 9: e1003809, 2013.

42. Rodon J, Dienstmann R, Serra V and Tabernero J: Development of PI3K inhibitors: lessons learned from early clinical trials. Nat Rev Clin Oncol 10: 143-153, 2013.

43. Qin L, Zeng H and Zhao D: Requirement of protein kinase D tyrosine phosphorylation for VEGF-A165-induced angiogenesis through its interaction and regulation of phospholipase Cgamma phosphorylation. J Biol Chem 281: 32550-32558, 2006. 